\title{
10 Kultur und Persönlichkeitsstörungen
}

\author{
Marcel G. Sieberer
}

\subsection{Einleitung: Persönlichkeit und kultureller Kontext}

Persönlichkeit und Kultur sind zwei dynamisch voneinander abhängige Systeme, deren wechselseitiges Verhältnis von verschiedenen Wissenschaftsdisziplinen, insbesondere klinischer Psychologie, Psychiatrie, Psychoanalyse oder Anthropologie, sehr unterschiedlich interpretiert wird. Der wissenschaftliche Diskurs über das Verhältnis von Kultur und Persönlichkeit wurde maßgeblich von einer zentralen Kontroverse um Kulturrelativismus und Kulturuniversalismus geprägt (Van de Vijver $u$. Leung 2001). Die kulturrelativistische Position vertrat dabei die Auffassung, dass Menschen, die in derselben kulturellen Umgebung aufwachsen und einen vergleichbaren Sozialisationsprozess durchlaufen, mit hoher Wahrscheinlichkeit und zu weiten Teilen dieselbe Persönlichkeit entwickeln. Die Natur des Selbst wird in diesem Sinne als sozial konstruiert und kulturvariant betrachtet, und die Existenz von relativ kulturunabhängigen Persönlichkeitsmerkmalen wird demzufolge infrage gestellt (Church 2000; Markus u. Kitayama 1998).

Die Vertreter des Kulturuniversalismus postulierten hingegen, dass grundlegende psychologische Bedingungen der menschlichen Natur und Persönlichkeit universell angelegt und von kulturellen Einflüssen weitgehend unabhängig sind (Poortinga u. Van Hermert 2001). Die Forschungsbemühungen gingen somit 
in erster Linie dahin, universelle Persönlichkeitsdimensionen und -prozesse $\mathrm{zu}$ identifizieren (Church 200o). Für das derzeit populärste dimensionale Modell, das Big-Five-Persönlichkeitsmodell, oder auch „Fünf-Faktoren-Modell“ (s.u.), konnte in Studien belegt werden, dass sich diese Dimensionen kulturübergreifend wiederfinden lassen (McCrae 2001; McCrae et al. 1998; Übersicht bei Church 2000, 2001). Viele Autoren halten heute eine integrierte Sichtweise beider Ansätze für richtig und vertreten die Auffassung, dass Persönlichkeitsfaktoren universell nachweisbar, aber kulturvariant ausgeprägt sind (Poortinga u. Van Hermert 2001; Church 2000).

Unter der Persönlichkeit eines Menschen wird die mehr oder weniger feste und überdauernde Organisation des Charakters, des Temperaments, des Intellekts und der Physis verstanden, die eine Entsprechung in individuellen, stabilen Verhaltenskorrelaten findet (Übersicht in Amelang u. Bartussek 1997). Jedes abstrahierbare und relativ konstante Persönlichkeitsmerkmal (engl. „trait“) prädisponiert für einzelne Verhaltensaspekte einer Person in bestimmten Situationen. In seinen Arbeiten über Kultur und Persönlichkeit hebt Foulks (1996) hervor, dass verschiedene Kulturen tendenziell unterschiedliche Persönlichkeitszüge als ideal erachten.

\section{Die Beurteilung der Abweichung einer Persönlichkeit von der sozial akzep- tierten Norm ist in hohem Maße von den Werten, Vorstellungen und Nor- men der jeweiligen Kultur und den sozialen Strukturen der Gesellschaft abhängig.}

Da die Übergänge von einer sozial akzeptierten in eine nicht akzeptierte $\mathrm{Ab}$ weichung von Persönlichkeitsmerkmalen sehr kontextabhängig und fließend sind, wird laut Fiedler (1998) „die Diagnose Persönlichkeitsstörung fast zwangsläufig in einem Bereich persönlicher und zwischenmenschlicher, wissenschaftlicher und gesellschaftlich-kultureller Streitfragen und Konfliktzonen“ gestellt. Dieser eher kulturrelativistischen Positionierung der kulturellen Psychiatrie stehen Studien gegenüber, die eine kulturübergreifende Anwendbarkeit der Konstrukte der Achse-II des DSM-IV zeigen. So haben beispielsweise Yang et al. (200o) das „Personality Diagnostic Questionnaire“(PDQ) und das „Personality Disorders Interview“ (PDI-IV) für eine chinesische Patientenpopulation ( $\mathrm{N}=2451)$ adaptiert und gezeigt, dass die Konzeptionen von Persönlichkeitsstörungen und die Kriterien des DSM-IV auf die chinesische Patientenpopulation mit ausreichender Reliabilität und Validität übertragbar waren. In der WHO-Studie von Loranger et al. (1994) wurde die Erhebung mit dem „International Personality Disorder Examination“ (IPDE), einem semistrukturierten Interview anhand der Kriterien des ICD-10 und des DSM, durchgeführt. Die Interviewer aller weltweit beteiligten Zentren konnten anhand des IPDE beide Klassifikationssysteme mit zufriedenstellender Interrater-Reliabilität auf ihre jeweilige Kultur und Sprache anwenden. 
In Abgrenzung zu den vorgenannten kategorialen Diagnosesystemen stellt der dimensionale Ansatz des Fünf-Faktoren-Modells zur Beschreibung von Persönlichkeitsstörungen eines der populärsten faktorenanalytisch begründeten Modelle dar, wonach Persönlichkeit im Wesentlichen auf fünf Einzelfaktoren zurückgeführt werden kann (vgl. Goldberg 1981; McCrae u. Costa 1987):

- Neurotizismus

- Extraversion

- Offenheit für Erfahrungen

- Gewissenhaftigkeit bzw. Rigidität

- Verträglichkeit

Die kulturübergreifende Anwendbarkeit und Gültigkeit des Fünf-FaktorenModells der Persönlichkeit ist in internationalen Studien inzwischen gut belegt (McCrae 2001). Untersuchungen bestätigten die Universalität und interkulturelle Validität des Fünf-Faktoren-Modells unter anderem für Chinesen, Deutsche, Israelis, Koreaner, Portugiesen (McCrae u. Costa 1997), Franzosen, Philippiner (McCrae et al. 1998) oder Finnen, Kanadier und Polen (Costa et al. 2002).

Die gegenwärtige Datenlage aus interkulturellen Studien spricht gleichzeitig für eine kulturell variierende Ausprägung einzelner Persönlichkeitsfaktoren. Beispielsweise zeigt sich in europäischen und amerikanischen Kulturkreisen im Vergleich zu asiatischen und afrikanischen eine stärkere Betonung der Persönlichkeitszüge Extraversion und Offenheit für Erfahrungen (Allik u. McCrae 2004). Zudem sind in asiatischen und europäischen Ländern im Vergleich zu afrikanischen oder amerikanischen Nationen höhere Werte für Neurotizismus beschrieben (McCrae 2002). Eine Studie mit interkulturell vergleichendem Ansatz hat als Hinweis auf die interkulturelle Variabilität von Persönlichkeit herausgearbeitet, dass philippinische Studenten verletzlicher, weniger emotional und gesprächig und dafür eher introvertiert waren als eine Gruppe amerikanischer Studenten (Huang et al. 1997). In einer weiteren interkulturell konzipierten Studie hatten Personen türkischer Herkunft im Vergleich zu denen deutscher Herkunft tendenziell höhere Werte für Extraversion und Gewissenhaftigkeit und eher niedrigere Werte für Neurotizismus, Offenheit für Erfahrungen und Verträglichkeit (McCrae 2002). Trotz dieser interkulturellen Variabilität der genannten Persönlichkeitsfaktoren variieren die Mittelwerte einzelner, wichtiger Persönlichkeitsfaktoren aber scheinbar stärker innerhalb einer Kultur als zwischen unterschiedlichen Kulturen (McCrae 2001; Allik u. McCrae 2004).

\subsection{Epidemiologische Aspekte}

Persönlichkeitsstörungen sind für die meisten Kulturen und Gesellschaften der Welt beschrieben. Es gibt allerdings nur wenige verlässliche epidemiologische Daten über Persönlichkeitsstörungen im interkulturellen Vergleich. 
Die bisherigen Studien zur Prävalenz spezifischer Persönlichkeitsstörungen in unterschiedlichen Kulturen sind darüber hinaus im Ergebnis uneinheitlich. So gibt es beispielsweise Hinweise darauf, dass die Prävalenz der Borderline-Persönlichkeitsstörung in den USA angestiegen ist (Millon 1993), während sie in vielen anderen Gesellschaften, vor allem in Asien, nicht entsprechend zunahm (Tseng 2001).

Die Forschung auf diesem Feld wird durch kulturspezifische Konzeptionen und Definitionen von Persönlichkeitsstörungen sowie durch kulturvariante Unterschiede hinsichtlich der sozialen Toleranz der jeweiligen sozialen Gemeinschaft gegenüber psychiatrischen Symptomen oder Verhaltensauffälligkeiten erschwert (vgl. Alarcon et al. 1998). Darüber hinaus macht eine erhebliche Heterogenität selbst innerhalb einzelner Ethnien eine Differenzierung der Prävalenz verschiedener Persönlichkeitsstörungen in bestimmten Bevölkerungsgruppen äußerst schwierig (Briken u. Kraus 200o). Ohne Kenntnis der jeweiligen kulturellen Bedeutung und Implikationen, der verwendeten Methoden und der diagnostischen Kriterien bleibt erhaltenes Datenmaterial darüber hinaus schwierig zu interpretieren. So wurde in Taiwan eine mit der Epidemiological Catchment Area Study (Nestadt et al. 1994) vergleichbare Studie durchgeführt, um die Prävalenzen der antisozialen Persönlichkeitsstörung in Amerika und Taiwan zu vergleichen (Compton et al. 1991). Die Ergebnisse zeigten, dass die Prävalenz für die antisoziale Persönlichkeitsstörung in Taiwan bei $0,2 \%$ lag, in den Vereinigten Staaten hingegen bei 3,0\%. Obwohl ähnliche methodische Instrumente verwendet werden und vergleichbare Studienpopulationen zugrunde lagen, ist nicht klar, ob die gewonnen Daten tatsächliche Unterschiede in der Prävalenzrate widerspiegeln. Vielmehr könnten kulturelle Unterschiede der gesellschaftlichen Bewertung bzw. der Reglementierung von antisozialem Verhalten zu einem Bias durch eher "sozial erwünschte“ Antworten der Befragten geführt haben (Tseng 2001).

Im Vergleich zu westlichen Ländern fallen in China die berichteten Prävalenzen von Persönlichkeitsstörungen ungewöhnlich niedrig aus (Tang $u$. Huang 1995). In der aktuellen Version des offiziellen chinesischen Klassifikationssystems, der Chinese Classification of Mental Disorders - Version 3 (CCMD-3), ist u.a. die Borderline-Persönlichkeitsstörung (BPS) als diagnostische Entität abermals nicht aufgenommen worden, obgleich Forschungsdaten durchaus dafür sprechen, dass die BPS auch in China zu den häufigeren Persönlichkeitsstörungen zählt (Zhong u. Leung 2009).

In der WHO-Pilotstudie von Loranger et al. (1994) kamen die unterschiedlichen Persönlichkeitsstörungen (gem. DSM-III-R) in praktisch allen teilnehmenden Ländern vor. In den meisten Ländern wurden die Borderline-Störung (14,5\%) und die selbstunsicher-vermeidende Persönlichkeitsstörung (11,0\%) am häufigsten diagnostiziert; lediglich aus Indien wurde keine vermeidende Persönlichkeitsstörung und aus Kenia keine Borderline-Persönlichkeitsstörung berichtet. Interessanterweise war selbst die konzeptuell umstrittene passiv-ag- 
gressive Persönlichkeitsstörung bei einer Häufigkeit von 5,0\% mit nur einer Ausnahme in allen teilnehmenden Ländern diagnostizierbar. Allerdings erlauben diese Ergebnisse keine direkten Rückschlüsse auf die tatsächlichen Prävalenzraten der jeweiligen Persönlichkeitsstörungen in der Allgemeinbevölkerung, da die Erhebungen größtenteils auf klinischen Populationen und damit nicht auf repräsentativen Stichproben basierten.

\section{Mithilfe der gegenwärtigen wissenschaftlichen Literatur lässt sich die Frage, ob spezifische Persönlichkeitsstörungen in unterschiedlichen Län- dern, Kulturen oder ethnischen Gruppen gleich häufig vorkommen, nicht beantworten.}

Die derzeitig verwendeten Klassifikationen und Konzepte von Persönlichkeitsstörungen sollten daher in zukünftigen Studien zunächst genauer auf ihre kulturelle Übertragbarkeit hin überprüft werden. In einem weiteren Schritt könnten dann aussagekräftige interkulturell vergleichende Studien sowie prospektive Untersuchungen an nicht-klinischen Populationen die Zusammenhänge von Kultur, Ethnie und Persönlichkeitspathologie besser aufklären.

\subsection{Kulturelle Faktoren der Ätiologie von Persönlichkeitsstörungen}

Persönlichkeitsstörungen sind nicht nur durch biologische Dispositionen, genetische Determinanten, Lern- und psychodynamische Vorgänge sowie biographisch gewachsene Haltungen bedingt, sondern auch durch den Einfluss sozialer und kultureller Faktoren gekennzeichnet. Nach Fabrega (1994) sind insbesondere biologische, historische und kulturelle Faktoren in ihrem dialektischen Zusammenspiel bei der Entwicklung von Persönlichkeitsstörungen bedeutsam.

Ein Aspekt, der im wissenschaftlichen Diskurs über die Ursachen, Symptomatik und Diagnostik von Persönlichkeitsstörungen bisher noch zu wenig Beachtung gefunden hat und der gleichzeitig zu einem besseren Verständnis der Ätiologie und der Behandlung von Persönlichkeitsstörungen beitragen könnte, ist der Einfluss von Kultur und Ethnizität (Tseng 2001). Kulturelle Faktoren können sich auf die Persönlichkeitsentwicklung einerseits protektiv, andererseits aber auch pathogen auswirken oder die Symptombildung pathoplastisch beeinflussen. Kultur kann dabei in zweierlei Hinsicht als pathogener Faktor fungieren: Zum einen können Elemente der mikrokulturellen (Familie) und der makrokulturellen Umgebung (Gesellschaft) die klinische Manifestation von Persönlichkeitsstörungen begünstigen. Zum anderen können Persönlichkeitsstörungen über kulturvariante Merkmale zur Symptomatik von Achse-I-Diagnosen (z.B. Depressionen) beitragen (Alarcon et al. 1998). Die pathoplastische Funktion kultureller Faktoren wird an dem Einfluss deutlich, den 
Tradition, Kindererziehung, Religion, Überzeugungen und Mythen, Normen und Werte und wirtschaftliche Situation auf der Makroebene sowie familiäre Regeln und Erwartungen, Traditionen der Ursprungsfamilie, Lebensumstände und signifikante Lebensereignisse auf der Mikroebene auf ein Individuum ausüben können.

Aktuelle internationale Untersuchungen aus den Niederlanden, Belgien und Australien weisen darauf hin, dass die Variabilität phänotypischer Merkmalsausprägungen bspw. der Borderline-Störung zu einem nennenswerten Anteil (> 40\%) auch auf additive genetische Einflüsse zurückgeführt werden können (Distel et al. 2008, 2009). Daraus lassen sich neue ätiopathogenetische Hypothesen generieren, insbesondere in Richtung von Gen-Umwelt-Interaktionen, deren Bedeutung für die Entstehung und Symptombildung von Persönlichkeitsstörungen noch weitgehend ungeklärt ist und deshalb auch ein Gegenstand zukünftiger interkultureller Untersuchungen sein sollte. Vor dem Hintergrund der zahlreichen kulturellen Einflussfaktoren und der phänotypischen Variabilität von Persönlichkeitsstörungen müssen gegenwärtige neurowissenschaftliche Interpretationen einzelner PKS als das Ergebnis einer gestörten Neurotransmission, wie beispielsweise der Borderline-Störung als eine Dysregulation des endogenen Opiod-Systems (vgl. Bandelow et al. 2010) allerdings einseitig und reduktionistisch erscheinen, zumindest solange die kulturellen Phänomene dabei unberücksichtigt und unerklärt bleiben.

\subsection{Interkulturelle Einflussgrößen bei der Diagnostik}

Kulturelle Einflussfaktoren spielen bei jedem Schritt des diagnostischen Prozesses eine bedeutsame Rolle: Dies wird schon in der Arzt-Patienten-Interaktion deutlich, bei der eine hohe Kultursensibilität notwendig ist, um zwischen Angehörigen verschiedener Ethnien überhaupt eine Basis für eine therapeutische Beziehung herzustellen (Mezzich 1995). Die Notwendigkeit einer kultursensiblen Herangehensweise konkretisiert sich beispielsweise an den Fragen, wie man in angemessener Weise Familienangehörige in die Anamneseerhebung mit einbezieht, wie man intime persönliche Details exploriert, wie man die Patientenangaben vor dem jeweiligen kulturellen Hintergrund im Hinblick auf Bedeutung und Schwere des seelischen Leidens interpretiert und wie man das soziale Funktionsniveau und das Ausmaß sowie den Wert sozialer Unterstützung bei Menschen aus anderen Kulturkreisen adäquat einschätzt (Mezzich et al. 2001).

Die diagnostische Einordnung von Persönlichkeitsstörungen hängt in entscheidendem Maße auch davon ab, wie eine Gesellschaft ein bestimmtes Verhalten bewertet. Daraus resultieren interkulturelle Unterschiede im diagnostischen Prozess, die zu Unter, aber auch Überbewertung kultureller Einflussfaktoren führen können. 
Im US-amerikanischen DSM-IV finden sich erstmals spezielle Abschnitte im Begleittext, die auf kulturspezifische Aspekte der jeweiligen Störungsbilder eingehen, um die Sensibilität für kulturabhängige Variationen im Ausdruck psychischer Störungen zu erhöhen und mögliche Folgen einer kulturell bedingten Fehleinschätzung im diagnostischen Prozess zu reduzieren. Als kulturspezifische Aspekte sind in erster Linie eine große sprachliche und auch somatische Variationsbreite in der Affektäußerung, ethnographische Unterschiede des kulturellen Kontextes und der Einfluss der Persönlichkeit auf kulturspezifische Ausdrucksformen hervorzuheben (Alarcon 1995). Im DSM-IV wird betont, dass bei der Diagnostik von Persönlichkeitsauffälligkeiten und Verhaltensstörungen der entsprechende soziokulturelle Kontext von entscheidender Bedeutung sei. Bei Menschen aus anderen Kulturkreisen müsse bedacht werden, inwieweit Sitten und Gebräuche, religiöse Vorstellungen und politische Überzeugungen aus der Ursprungskultur zum Ausdruck kommen. Darüber hinaus macht der Begleittext des DSM-IV zu den Persönlichkeitsstörungen deutlich, dass diese differenzialdiagnostisch nicht verwechselt werden dürfen mit Problemen, die im Zusammenhang mit der Akkulturation bei Migration entstehen. Allerdings sind wichtige Aspekte kultureller Dimensionen wie Selbstbild, Anpassung oder sozialer Kontext auch im DSM-IV bisher weitgehend vernachlässigt worden (Alarcon 1996).

\subsection{Kulturelle Aspekte spezifischer Persönlichkeitsstörungen}

Eine Einordnung einzelner Persönlichkeitsmerkmale und überdauernder Verhaltensmuster in den jeweiligen soziokulturellen Bezugsrahmen ist notwendig, um normalpsychologisches Erleben, Fühlen und Handeln von den psychopathologischen Merkmalen einer Persönlichkeitsstörung diagnostisch abgrenzen zu können. In den folgenden Abschnitten werden für die einzelnen spezifischen Persönlichkeitsstörungen die kulturellen Aspekte, die für die Beurteilung des Funktionsniveaus einer Persönlichkeit und für eine kultursensible psychopathologische Einordnung zu berücksichtigen sind, herausgearbeitet und in Anlehnung an die Systematik des ICD-10 im Einzelnen dargestellt. Die interkulturelle Persönlichkeitsforschung hat bisher am ausführlichsten die dramatisch und emotional imponierenden Persönlichkeitsstörungen (PKS) des Cluster B berücksichtigt, d.h. vor allem die emotional-instabile, die histrionische und die dissoziale PKS sowie die gemäß DSM-IV definierte narzisstische Persönlichkeitsstörung.

Trotz der weltweiten Verbreitung der emotional-instabilen Persönlichkeitsstörung vom Borderline Typ (ICD-10: F6o.31) existieren bisher nur wenige interkulturelle Untersuchungen $\mathrm{zu}$ phänomenologischen und psychopathologischen Unterschieden der Borderline-Persönlichkeitsstörung (BPS) in unterschiedlichen Kulturen und Gesellschaften. Eine erste klinische Studie zur BPS in Asien ergab, dass japanische Borderline-Patienten häufiger unter Derealisations- und Depersonalisationserleben litten und seltener einen komorbiden 
Substanzmissbrauch zeigten, sich sonst aber klinisch nicht wesentlich von den amerikanischen Borderline-Patienten unterschieden (Moriya et al. 1993; Ikuta et al. 1994). Depersonalisationserleben, tranceähnliche Zustände und psychosenahe Episoden sind allerdings in vielen nicht-westlichen Gesellschaften bekanntermaßen auch kulturelle Phänomene, die im Rahmen von traditionell verwurzeltem, religiösen Erleben zu finden sind und sich unter dem Anpassungsdruck an eine neue kulturelle Umgebung vorübergehend verstärken können (Kirmayer 1989; Lewis-Fernandez 1993). Die sozialen Anforderungen an den Einzelnen sind in modernen Lebenswelten um ein vielfaches gestiegen, sodass Identitäts- und Anpassungsfragen elementare Bedeutung erlangen ( $\mathrm{Pa}-$ ris 1991). Ungelöste intrapsychische Konflikte können zu Stimmungsschwankungen, Impulsivität, Instabilität, Ambivalenz, Unberechenbarkeit und intensivem Ärger führen, was nicht zwanglos mit einer BPS gleichgesetzt werden darf. Überdies hebt der DSM-IV hervor, dass Jugendliche in der Adoleszenz, insbesondere im Zusammenhang mit Substanzmissbrauch, vorübergehende Phasen von emotionaler Instabilität, Unsicherheit und Ambivalenz sowie konflikthafter sexueller Orientierung durchleben können, die durchaus an Borderline-Symptome erinnern können (APA 200o). Zudem sind bei der diagnostischen Einordnung suizidalen Verhaltens im transkulturellen Kontext die teils erheblich variierenden ethisch-moralischen Bewertungen von Suizidalität oder der Einfluss von Suizidtraditionen in den verschiedenen Kulturen differenziert zu berücksichtigen (Calliess et al. 2007). Auch parasuizidale Handlungen, wie beispielsweise Schnittverletzungen am Handgelenk, sind in Kulturen des Mittleren Ostens sowie bei indianischen Kulturen häufig Teil kulturell determinierter Bindungsrituale (Paris 1991). Andererseits beobachtete Paris (1992), dass gerade die hervorstechenden Symptome der Borderline-Persönlichkeitsstörung, i.e. selbstverletzendes Verhalten oder wiederholte Suizidversuche, in traditionellen Kulturen offenbar störungsassoziiert so nicht vorkommen.

Die dissoziale Persönlichkeitsstörung (ICD-10: F6o.2) zeigt auf der Symptomebene (bspw. hinsichtlich Impulsivität) eine enge Beziehung zur BorderlineStörung. In Gesellschaften mit einer hohen sozialen Kohäsion kommt sie laut Paris (1996) wahrscheinlich seltener vor. Allerdings existiert in den meisten Kulturen in irgendeiner Form ein Begriff für die anti- bzw. dissoziale Persönlichkeit (Cooke 1996). In einer kulturvergleichenden Untersuchung aus Mitte der 1970er-Jahre haben selbst so unterschiedliche Ethnien wie die Inuit im Nordwesten von Alaska und die Yoruba in Nigeria eigene Konzepte für sozial abweichendes Verhalten gezeigt (Murphy 1976). Das Vorkommen der dissozialen Persönlichkeitsstörung scheint prinzipiell unabhängig von dem sozioökonomischen Status einer Gesellschaft, wobei die tatsächliche Prävalenz abhängig von Zeit und Ort variieren kann (Robins et al. 1991). Cooke und Michie (1999) formulieren als zentrale These ihrer Arbeiten, dass in kollektivistischen Kulturen, in denen die Bedürfnisse der sozialen Gemeinschaft Vorrang vor individuellen Zielen haben, wo Autoritäten eher respektiert werden und 
stabile, langfristige Beziehungen bedeutsam sind, die Kriminalitätsraten geringer ausfallen als in individualistischen Gesellschaften. Letztere leisteten aufgrund starken Konkurrenzdenkens und Leistungsstrebens sowie aufgrund von Autoritätsverlust und Individualisierung mit geringer sozialer Bezogenheit, Vereinzelung und oberflächlichen Beziehungen einem rücksichtslosen, manipulativen und ausbeuterischen oder auch kriminellen Verhalten Vorschub. Andererseits kann in bestimmten gesellschaftlichen Kontexten politisch oppositionelles oder jugendliches Protestverhalten als mangelhafte Anpassung an bestehende Normen und somit als sozial abweichendes Verhalten fehlinterpretiert werden: Alarcon und Foulks (1995) hoben in diesem Kontext hervor, dass bei Angehörigen von jugendlichen Subgruppierungen (engl. „gangs“) die antisoziale Persönlichkeitsstörung in etwa der Hälfte der Fälle fehldiagnostiziert sein könnte, da die diagnostischen Kriterien in einem Milieu, in dem Gewalt als Überlebensstrategie erlernt wird, nicht ohne Weiteres greifen.

Eine weitere Persönlichkeitsstörung des Cluster B, bei der ethnozentristische Perspektiven häufiger zu Fehlinterpretationen führen, ist die histrionische Persönlichkeitsstörung (ICD-10: F6o.4). In einigen Kulturen sind in der Spätadoleszenz und im frühen Erwachsenenalter kulturell determinierte Verhaltensvarianten zu beobachten, die sich durch Hyperemotionalität, Selbstzentriertheit, Charme, Verführbarkeit, Beeindruckbarkeit, Unterwürfigkeit, Somatisierung und eine Tendenz zur Dramatisierung auszeichnen können und unter Beachtung des jeweiligen kulturellen Bezugsrahmens als normalpsychologisch zu bewerten sind (Standage et al. 1984). Im interkulturellen Setting werden Menschen aus dem Mittelmeerraum oder aus Lateinamerika wahrscheinlich am häufigsten mit einer histrionischen Persönlichkeitsstörung fehldiagnostiziert. Andererseits haben Castaneda und Franco (1985) darauf hingewiesen, dass kulturfremde Diagnostiker auch in anderer Richtung der Gefahr kultureller Stereotypien unterliegen: In ihrer Untersuchung ordneten amerikanische Interviewer bei Patientinnen lateinamerikanischer Abstammung Symptome wie histrionisches Verhalten, eine geringe Affektkontrolle und Impulsivität häufiger fälschlicherweise als kulturtypisch denn als psychopathologisch ein.

Die narzisstische Persönlichkeitsstörung wird gemäß DSM-IV auch dem Cluster B zugerechnet und als eigenständige Diagnose geführt (DSM-IV: 301.31), während sie im ICD-1o nur unter sonstige spezifische Persönlichkeitsstörungen (ICD-10: F6o.8) verschlüsselt werden kann. Es wird vermutet, dass narzisstische Persönlichkeitsstörungen bei ethnischen Minderheiten häufig fehldiagnostiziert werden, insbesondere bei denen, die der Aufnahmekultur sehr fremd sind (Tseng 2001). Flamboyance oder „Extravaganz“ im Sinne einer übertriebenen Bewertung der eigenen Wichtigkeit wird beispielsweise bei jungen Männern in südeuropäischen oder lateinamerikanischen Kulturen häufiger beobachtet und als narzisstisch fehlinterpretiert (Martinez 1993). Eine wortreiche, blumige Ausdrucksweise kann als Rationalisierung und das Bestreben, 
institutionelle hindernde Umstände selbst beheben zu wollen, als Größenphantasie verkannt werden (APA 200o). Mitglieder von sogenannten GhettoGruppen, bei denen das Selbstwertgefühl häufiger fragil ist, können Verhaltensweisen zeigen, die durch eine pseudonarzisstische Enttäuschung und Kränkung geprägt sind (Battan 1983) und damit bei isolierter Betrachtung als narzisstisch strukturierte Persönlichkeiten anmuten.

Die im Cluster A zusammengefassten, sonderbar und exzentrisch anmutenden paranoiden und schizoiden PKS haben in der interkulturellen Persönlichkeitsforschung bisher weniger Beachtung gefunden.

Für die paranoide Persönlichkeitsstörung (ICD-10: F6o.o) sind einige ältere Studien zu berücksichtigen, die darauf hinwiesen, dass manche ethnische Gruppen kulturell determinierte Verhaltensweisen an den Tag legen, die als paranoid fehleingeschätzt werden könnten. Beispielsweise würden Menschen aus dem Mittleren Osten oder aus Osteuropa Außenstehenden gegenüber eher geheimnistuerisch und misstrauisch wirken (Trouve et al. 1983) oder Südeuropäer würden häufiger den Eindruck erwecken, als seien sie etwa rechthaberisch oder bezüglich gesellschaftlicher Normen weniger anpassungsbereit (Padilla et al. 1987). Migranten, Flüchtlinge oder Angehörige ethnischer Minderheiten, können zudem aufgrund traumatisierender Erlebnisse im Herkunftsland, aufgrund einer Sprachbarriere oder einer mangelnden Vertrautheit mit den Werten und Normen der Aufnahmekultur oder erfahrener Ablehnung im Aufnahmeland mit extremer Vorsicht, hoher Anspannung oder Misstrauen reagieren. Häufig ist die Fähigkeit zur freien Entscheidungsfindung bei diesen Personen eingeschränkt, was sie rigide und nur wenig kompromissfähig erscheinen lassen mag und in einen circulus vitiosus wechselseitigen Misstrauens mit den Mitgliedern der Aufnahmekultur münden kann. Streitsüchtiges Verhalten, Misstrauen und eine übertriebene Empfindlichkeit stellen formal zwar Kernkriterien der paranoiden Persönlichkeitsstörung dar, müssen in dem geschilderten Kontext hinsichtlich ihrer diagnostischen Wertigkeit jedoch relativiert werden (Alarcon et al. 1998).

Über die schizoide Persönlichkeitsstörung(ICD-10: F6o.1) liegen bis dato keinerlei aussagekräftigen interkulturellen Studien vor. Dabei ergeben sich gerade für Menschen, die in ein fremdes Land emigrieren oder die einen durchgreifenden gesellschaftlichen Wandel oder Umbruch persönlich erfahren, Konstellationen, die zu Reaktionsweisen disponieren, die an schizoides Verhalten erinnern können und von diesem abzugrenzen sind. So können beispielsweise Menschen, die vom Land in die Stadt gezogen sind, mit einer länger dauernden Phase emotionalen Rückzugs und sozialer Isolation reagieren, bis die Anpassungsleistung vollbracht ist. Einzelgängerisches Verhalten, Affekthemmung und Mangel an vertrauensvollen Beziehungen sind in diesen Lebenssituationen eher als Verarbeitungsmodus denn als Kriterien für eine schizoide Persönlichkeitsstörung zu bewerten. Analog können auch Zuwanderer aus fremden Kulturen in diesem Sinne von Mitgliedern der Aufnahme- 
gesellschaft als zurückgezogen, feindselig, kalt oder gleichgültig erlebt werden. Systematische Studien über diese Persönlichkeitsstörung mit einem kulturvergleichenden bzw. interkulturellen Ansatz stehen wie gesagt aber noch aus.

Auch zu den PKS des Cluster C, die durch ängstliche und vermeidende Verhaltensweisen geprägt sind, liegen bisher nur wenige und deskriptiv angelegte wissenschaftliche Daten vor. Für die anankastische Persönlichkeitsstörung (ICD-10: F6o.5) erscheint eine zuverlässige diagnostische Bewertung eines bestimmten Verhaltens ohne Kenntnis des soziokulturellen Hintergrunds besonders schwierig. So kann die Ausübung von Regeln und Vorschriften, die einem Individuum durch den jeweiligen kulturellen Bezugsrahmen auferlegt werden kann, den Eindruck mangelnder Flexibilität und Toleranz hervorrufen und dadurch an eine zwanghafte Persönlichkeitsstörung erinnern. Angehörige bestimmter, gesellschaftlich-kulturell hervorgehobener Gruppen, wie z.B. religiöse Autoritäten, ranghohe Politiker, Beamte oder Wissenschaftler, zeigen zuweilen einen eingeschränkten Affektausdruck, wirken in moralischen und ethischen Fragen rigide und haben ein erhöhtes Kontrollbedürfnis, wodurch es auch zu Konflikten mit den weniger rigiden Teilen einer Gesellschaft kommen kann (Alarcon et al. 1998; Witztum et al. 1990). Ein erhöhtes Risiko der Fehldiagnose einer anankastischen PKS besteht somit bei religiösem Konservativismus oder bei starker Anpassung an enge soziale Regeln. Exemplarisch seien dazu die religiösen Vorschriften und Praktiken orthodoxer Juden oder die Arbeitshaltung japanischer Arbeitnehmer genannt (Chang 1995). In demselben Sinne mögen Europäer auf andere Kulturkreise häufig ordentlich, vorsichtig, unflexibel, affektarm und formell erscheinen, was eine kulturell geprägte Komponente ihrer Gesellschaften widerspiegelt und nicht grundsätzlich mit der Diagnose einer zwanghaften Persönlichkeitsstörung in Zusammenhang gebracht werden sollte (DeGregorio u. Carver 1980).

Strenge religiöse Praktiken, familienzentrierte Werteorientierung und identitätswahrende Prozesse können in der transkulturellen Diagnostik irrtümlicherweise auch den Eindruck einer ängstlich-vermeidenden Persönlichkeitsstörung (ICD-10: F6o.6) vermitteln (Roland 1988): Kulturell tief verwurzelte Sitten, Werte und Gebräuche können ein Individuum neben einem hohen Bedürfnis nach Akzeptanz und Integration scheu und isoliert erscheinen lassen. Solches Verhalten wird häufiger für Asiaten und Philippinern beschrieben und kann fälschlicherweise als Inferioritätskomplex interpretiert werden. Auch bei Migranten kann durch Probleme im Akkulturationsprozess ein vermeidendes, selbstunsicheres Verhalten auftreten (APA 200o). Angehörige ethnischer Minderheiten sind in bestimmten sozialen Situationen eher zurückhaltend und im Kontakt mit anderen Menschen ängstlich, fürchten Kritik und können insgesamt nur schwer mit Angehörigen anderer ethnischer Cruppen in Kontakt treten. Solches Verhalten im Rahmen der Akkulturation ist von pathologisch gesteigertem ängstlich-vermeidendem Verhalten abzugrenzen. 
Auch für die abhängige Persönlichkeitsstörung (ICD-10: F6o.7) ist zu berücksichtigen, dass in asiatischen Gesellschaften im Gegensatz zu westlichen Kulturen Passivität, Höflichkeit und Abhängigkeit erwünschte Persönlichkeitszüge darstellen (Roland 1988; Johnson 1993). Auch heutzutage spiegeln Gehorsam und Gefolgschaft gegenüber Älteren, Professionellen, religiösen Autoritäten oder Politikern teilweise noch die strengen Hierarchien und paternalistischen Strukturen in diesen Gesellschaften wider. Es ist daher auch bemerkenswert, dass die abhängige Persönlichkeitsstörung im chinesischen Klassifikationssystem psychischer Störungen (CCMD) weiterhin gar nicht aufgeführt wird (Lee 1996).

Die vorstehend herausgearbeiteten kulturellen Aspekte von Persönlichkeitsstörungen verdeutlichen, dass eine korrekte Einordnung einzelner Persönlichkeitsmerkmale und Verhaltensmuster in den jeweiligen soziokulturellen Kontext unabdingbar ist, um normalpsychologisches Erleben, Fühlen und Handeln von psychopathologischen Merkmalen einer Persönlichkeitsstörung differenzieren zu können.

\subsection{Migration, Akkulturation und Persönlichkeitsstörungen}

\subsubsection{Soziokulturelle und migrationsspezifische Faktoren}

Interkulturelle Erfahrungen, wie sie durch eine Migration und den Akkulturationsprozess gemacht werden, können unter bestimmten Konditionen zur Entwicklung und zum Ausdruck von Symptomen einer Persönlichkeitsstörung beitragen (Krause 1998). Als mögliche Stressoren, die mit einer Migration verbunden sein können, sind u.a. Erfahrungen von Diskriminierung (Igel et al. 2010), das Phänomen des Rassismus (Fernando 1991) und vor allem der vielschichtige Prozess der Akkulturation (Fernando 1995; Krause 1998) zu nennen. Der Migrationsprozess kann als kulturelles, soziales und geographisches Geschehen wegen der damit einhergehenden Vulnerabilität die Entwicklung von Persönlichkeitsstörungen begünstigen (Alarcon et al. 1998). Paris (1996, 1998) hat für die emotional instabilen Persönlichkeitsstörungen herausgearbeitet, dass die Lebensbedingungen in traditionellen Gesellschaften soziale und kulturelle protektive Faktoren aufweisen, die trotz biologisch determinierter Prädisposition die Entwicklung einer Persönlichkeitsstörung verhindern können. Traditionelle Gesellschaften sind nach dieser Lesart charakterisiert durch einen langsamen sozialen Wandel, Kontinuität zwischen den Generationen, eine hohe familiäre und gesellschaftliche Kohäsion und eindeutige soziale Rollen (Paris 1998). Obwohl es große Unterschiede zwischen den einzelnen traditionellen Gesellschaften gibt, ist ihnen allen gemeinsam, dass klare Erwartungen an und eindeutige und vorhersagbare soziale Rollen für die Mitglieder einer solchen kulturellen Gemeinschaft existieren. Im Ergebnis werden vulnerable Individuen davor geschützt, sich nutzlos oder sozial isoliert zu 
fühlen. In modernen Gesellschaften hat der Wegfall stabiler sozialer Strukturen hingegen zu einem rapiden gesellschaftlichen Wandel mit einem Verlust sicherer sozialer Rollenmodelle geführt. Einzelne Autoren interpretieren die in den letzten Jahrzehnten vermutlich steigende Prävalenz von BorderlinePersönlichkeitsstörungen in westlichen Kulturen im Kontext der soziokulturellen und psychosozialen Auswirkungen eben dieses gesellschaftlichen Wandels (Paris 1996; Millon 200o). Man spricht in solchen Zusammenhängen auch von soziokultureller Desintegration (Leighton et al. 1963 zitiert nach Paris 1996). Letztlich können einzelne Persönlichkeitsstörungen von diesem Standpunkt aus als ein kulturelles Phänomen begriffen werden (Alarcon u. Leetz 1998).

Bei der Migration von einer traditionellen in eine moderne Gesellschaft wird der historische und soziale Prozess der Modernisierung Teil einer persönlichen Erfahrung. Die postmigratorischen Phasen der kritischen Anpassung, der Trauer und der generationsübergreifenden Anpassungsprozesse verändern die Identität der betroffenen Individuen selbst und gehen mit einer Entwicklungsphase einher, die sich als dritte Individuation oder als kulturelle Adoleszenz im Leben eines Menschen bezeichnen lässt (Machleidt 2007a; Machleidt u. Heinz 2008). Das Verlassen des eigenen Kulturraumes erfordert ähnlich wie in der Adoleszenz die Ablösung von den kulturtypischen Beziehungsobjekten, die als mütterliche bzw. väterliche Ersatzobjekte (z.B. Muttersprache und Vaterland) Surrogatcharakter haben. In der Adoleszenz wird das vertraute Drinnen der Familie verlassen und draußen erfolgt die Aufnahme in die Gesellschaft. Bei der Migration wird das vertraute Drinnen der Ursprungsgesellschaft verlassen und draußen wird die Aufnahme in eine fremde Kultur und Gesellschaft angestrebt. Allerdings vollzieht sich diese kulturelle Adoleszenz bei postadoleszenten Erwachsenen auf einem höheren und damit komplexeren Strukturniveau (Machleidt 2009). Migranten sind im Migrationsprozess vor allem in der Phase der kritischen Integration, d.h. innerhalb des Zeitabschnitts, in dem die Akkulturation als die wesentliche Integrationsleistung im Migrationsprozess erbracht wird, einer erhöhten Vulnerabilität für die Entwicklung von psychischen Störungen ausgesetzt (Machleidt 2007; Machleidt u. Calliess 2005).

Aktuelle Untersuchungen zum Verhältnis von Persönlichkeit und Kultur stützen die sogenannte Personality-Culture Clash Hypothese. Demzufolge wird die Pathogenität bestimmter Persönlichkeitszüge entscheidend durch den Grad der Gegensätzlichkeit der individuellen Persönlichkeitskonstitution und der soziokulturellen Ausrichtung der Umgebung bestimmt: So ist beispielsweise für Individuen mit kollektivistisch geprägter Persönlichkeitsstruktur bei Konfrontation mit individualistisch ausgerichteten Grundwerten einer Gesellschaft das Risiko für pathologische Ausprägungen einzelner Persönlichkeitszüge besonders erhöht (Caldwell-Harris u. Ayçiçegi 2006). 


\subsubsection{Prävalenz von Persönlichkeitsstörungen bei Migranten}

Eine US-amerikanische Längsschnittstudie kam zu dem Ergebnis, dass für die Gruppe der Hispanos eine höhere Prävalenz für Borderline-Persönlichkeitsstörungen anzunehmen ist als für die weißen oder afroamerikanischen Studienteilnehmer (Chavira et al. 2003). Ein erst kürzlich publiziertes systematisches Review mit einer Metaanalyse verfügbarer Quellstudien fand zwar signifikante Prävalenzunterschiede für Persönlichkeitsstörungen zwischen schwarzen und weißen Ethnien (OR 0.476, Cls 0.248-0.915, p = 0.026), konnte diese aber nicht zwischen Asiaten oder Hispanoamerikanern und Weißen feststellen (McGilloway et al. 2010). Andererseits ergab eine Untersuchung aus Spanien, dass Migranten im Vergleich zu einheimischen Patienten in einer psychiatrischen Notaufnahme seltener die Diagnose einer Borderline-Störung erhielten (Pascual et al. 2008). Insbesondere die Migranten aus Subsahara-Afrika (7-fach geringeres Risiko) oder aus Asien (4-fach geringeres Risiko) wiesen deutlich geringere Prävalenzraten für eine BPS auf als Migranten aus Südamerika, Nordafrika oder westlichen Herkunftskontinenten wie Europa, Nordamerika oder Australien. Neben einer Reihe methodischer Limitationen dieser Studie könnten mögliche Erklärungen für diese Beobachtung sein, dass die untersuchten Migranten erst kurze Zeit mit der Aufnahmekultur konfrontiert waren, dass Personen, die eine Migration vollziehen, insgesamt eine resilientere Persönlichkeit besitzen (healthy migrant effect) oder aber, dass interkulturelle Verzerrungseffekte bei der Diagnostik eine Rolle spielen. So hatten in der Untersuchung von Pascual et al. (2008) gerade die Migranten mit der größten Sprachbarriere das geringste Risiko, die Diagnose einer BPS zu erhalten. Ähnlich lautend ergab eine schwedische Studie zum Einfluss von Ethnizität auf psychiatrische Diagnosen bei ambulanten Patienten, dass bei den einheimischen Schweden ungefähr doppelt so häufig die Diagnose einer Persönlichkeitsstörung gestellt wurde wie bei Patienten mit anderer ethnischer Zugehörigkeit (Al-Saffar et al. 2004).

Die Ergebnisse einer unlängst durchgeführten eigenen Untersuchung zur Prävalenz der Borderline-Störung bei Migranten sprechen dafür, dass diese Persönlichkeitsstörung bei Patienten mit Migrationshintergrund in (teil-)stationärer psychiatrischer Behandlung seltener vorliegt bzw. seltener diagnostiziert wird als bei den einheimischen Patienten (Nielsen et al. 2011). Während in der Gruppe der einheimischen Patienten $(n=2120)$ in $6,5 \%$ der Behandlungsfälle eine BPS diagnostiziert wurde, fiel die Quote in der Gruppe der Patienten mit Migrationshintergrund im gleichen Zeitraum mit 3,5\% signifikant niedriger aus $\left(\mathrm{Chi}^{2}=5.02, \mathrm{df}=1, \mathrm{p}=0.025\right)$. Da es sich bei dieser Studie um eine retrospektive Analyse von Behandlungsdaten handelt, lassen diese Ergebnisse allerdings keine Rückschlüsse auf kausale Zusammenhänge und auch keine zuverlässigen Aussagen über tatsächliche Prävalenzraten zu. 


\subsubsection{Migration, Traumatisierung und Persönlichkeitsstörung}

Bei traumatisierten Migranten und Flüchtlingen können zusätzliche differenzialdiagnostische Probleme bei der Beurteilung einzelner Persönlichkeitsdimensionen auftreten, da Komorbiditäten mit bzw. Abgrenzungen zu etwaigen psychischen Traumafolgestörungen eine Rolle spielen. Schätzungen zufolge leiden bis zu 30\% der Flüchtlinge in der BRD aufgrund von Traumatisierung durch organisierte Gewalt unter psychischen Störungen (Brune 200o). Eigenen Untersuchungen zufolge liegt dieser Prozentsatz zumindest bei bestimmten Gruppen Asylsuchender sogar noch deutlich höher (Sieberer et al. 2011). Die Situation von Flüchtlingen und Migranten wird über eventuelle Gewalterfahrungen in den Herkunftsländern hinaus durch den Prozess der Migration, der ebenfalls potenziell traumatisierend sein kann sowie durch die Exilproblematik zusätzlich belastet. Hinzu treten psychosozial schwierige Rahmenbedingungen, beispielsweise durch einen unsicheren Aufenthaltsstatus.

Anhaltende dysphorische Verstimmungen, eine chronische Beschäftigung mit Suizidideen, eine Neigung zu Selbstverletzungen, explosive oder extrem unterdrückte Wut und zwanghafte oder äußerst gehemmte Sexualität, aber auch dissoziative Episoden, Depersonalisations- und Derealisationserleben sowie der Abbruch von intensiven Beziehungen machen eine Abgrenzung des komplexen postraumatischen Belastungssyndroms von der Borderline-Störung dann sehr schwierig, wenn nicht gar manchmal unmöglich (Bronisch 2001; Gunderson u. Sabo 1993). Die Wechselbeziehungen von Traumaerfahrung und Persönlichkeit sowie weitere wesentliche Einflussfaktoren für die Entstehung und Diagnostik von Persönlichkeitsstörungen bei Menschen mit Migrationserfahrung sind in Abbildung 43 komprimiert dargestellt. Das abgebildete in-

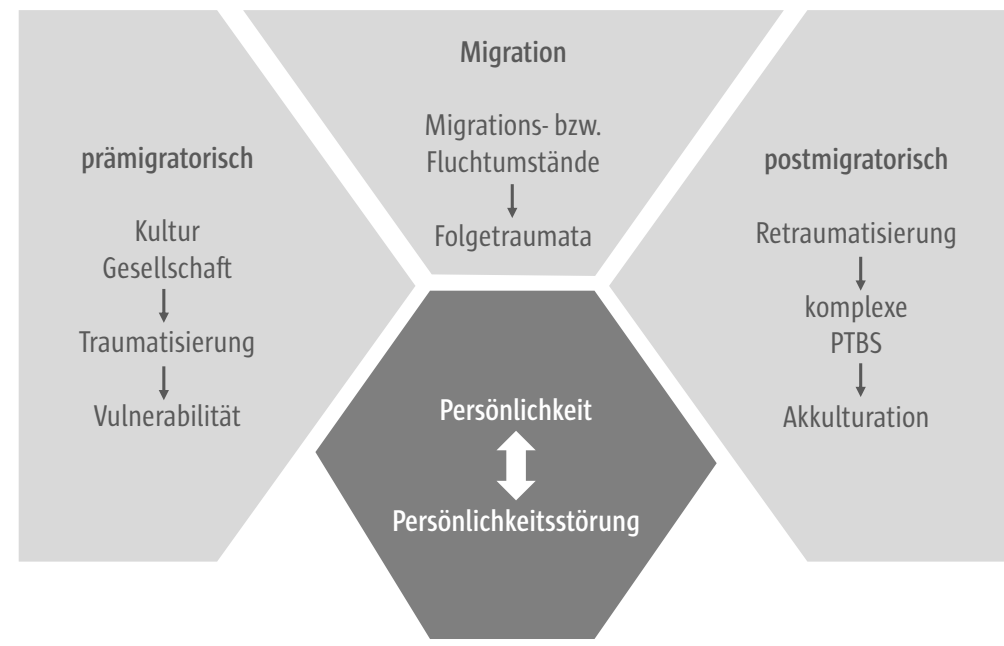

Abb. 43 Interaktionales Modell der Zusammenhänge von Migration, Trauma und Persönlichkeit (weiterentwickelt nach Calliess et al. 2008) 
teraktionale Modell veranschaulicht dabei die unterschiedlichen Zusammenhangsebenen von Migration, Persönlichkeit bzw. Persönlichkeitsstörung und verdeutlicht gleichzeitig die Rolle, die Traumatisierungen zu den unterschiedlichen Zeitpunkten des Migrationsprozesses für die Entwicklung von Persönlichkeitsstörungen und die daraus resultierenden differential-diagnostischen Probleme spielen können.

Neben den weiter oben ausgeführten kulturell-gesellschaftlichen Einflussfaktoren und dem Einluss einer multifaktoriellen Prädisposition bzw. einer individuellen Vulnerabilität können prämigratorische Traumatisierungen die Entwicklung von Persönlichkeitsstörungen begünstigen und gleichzeitig die Bewältigung der Migrationsanforderungen und damit letztlich das resultierende Akkulturationsergebnis beeinträchtigen. Auch traumatisierende Erfahrungen im Zusammenhang mit dem eigentlichen Migrationsakt, wie z.B. im Rahmen von Vertreibung und Flucht, können sich in gleicher Weise auf die Persönlichkeit auswirken. Vor allem bei vorbestehenden, prämigratorischen Traumatisierungen erhöht sich für den Fall einer Retraumatsierung zu einem späteren Zeitpunkt des Migrationsprozesses das Risiko, dass sich komplexe posttraumatische Störungsbilder entwickeln, die klinische Überlappungsbereiche mit Persönlichkeitsstörungen zeigen können oder von diesen differenzialdiagnostisch abgegrenzt werden müssen.

Eine prämigratorische Persönlichkeitsstörung kann als Vulnerabilitätsfaktor die Verarbeitung traumatisierender Erfahrungen, z.B. durch „man-made disasters" oder bestimmte migrationsspezifische Aspekte, negativ beeinflussen.

Postmigratorisch können schwere Traumatisierungen und Identitätskrisen ihrerseits eine Persönlichkeitsstörung induzieren bzw. verschlimmern oder ein komplexes posttraumatisches Belastungssyndrom hervorrufen, den Akkulturationsprozess erschweren oder gar die Ausbildung einer bi- oder multikulturellen Identität verhindern.

\subsection{Interkulturelle Aspekte der Therapie}

Im Hintergrund des Krankheitsgeschehens von psychischen Störungen bei Migranten lässt sich häufig eine migrations- bzw. kulturspezifische Problematik eruieren, die im Ergebnis die Krankheitsverarbeitung und -bewältigung stark beeinflussen kann. Zu den wesentlichen migrationsspezifischen Faktoren zählen u.a. ein erschwerter Kontakt zu nahe stehenden Menschen im Heimatland, aufenthaltsrechtliche Fragen, der sozioökonomische Status, mögliche Rückkehrabsichten sowie Verständigungsschwierigkeiten. Diese sind zu unterscheiden von kulturspezifischen Faktoren, wie z.B. divergente Traditionen und Rituale, verschiedene Werte und Normen oder andersartige 
Verhaltensmuster und Rollenerwartungen sowie Unterschiede hinsichtlich des Krankheitserlebens, der Krankheitskonzepte oder der Erwartungen an professionelle Helfer. Um zu verstehen, in welcher Weise und in welchem Ausmaß die Migrationsbiographie mit der Entwicklung seelischer Krisen zusammenhängt, muss bedacht werden, dass den Herausforderungen des Migrationsprozesses und der Akkulturation individuell sehr unterschiedlich begegnet werden kann.

Allgemein stellen psychotherapeutische Verfahren in der Therapie von Persönlichkeitsstörungen nicht nur eine weit verbreitete, sondern wohl auch die am besten untersuchte und die nachhaltig wirkungsvollste Behandlungsmethode dar. Alle Psychotherapieverfahren hängen von impliziten Modellen des Selbst ab, welche wiederum auf kulturellen Konzepten der Person fußen. Die zugrundeliegenden Konzepte von der Person bauen in den meisten Psychotherapieformen auf den euroamerikanischen Werten des Individualismus auf und kontrastieren mit den eher sozio-, umwelt- oder kosmozentrischen Ausrichtungen anderer Kulturen. Interkulturelle Psychotherapie muss daher das jeweilige kulturell determinierte Konzept von der Person im therapeutischen Prozess berücksichtigen und mit den Wertevorstellungen und dem kulturell begründeten Lebensstil des Patienten abgleichen (Kirmayer 2007).

In der psychiatrisch-psychotherapeutischen Behandlung von Migranten ist ein Perspektivenwechsel der Therapeuten von einer ethnozentristisch geprägten Sichtweise zu einer interkulturellen Erweiterung des eigenen Fachwissens erforderlich. Probleme im interkulturellen therapeutischen Prozess können rein sprachlicher Natur sein oder sich auf unterschiedliche Krankheitskonzepte und Rollenerwartungen von Patient und Therapeut beziehen (Brucks 2004; Tuna u. Salman 1999). Hier besteht das Risiko, diese Besonderheiten der Behandlung entweder zu negieren bzw. in ihrem Einfluss zu unterschätzen oder ihnen eine $\mathrm{zu}$ hohe Bedeutung beizumessen: In letzterem Fall werden dann jegliche Schwierigkeiten, die bei Migranten zu Symptomen führen oder in der Behandlung von Migranten auftreten, als „interkulturelle Konflikte“ gedeutet (Nika u. Baskedis 200o). Gerade die Verzahnung kultureller und migrationsbedingter Einflussfaktoren mit davon unabhängigen Größen in der Entwicklung seelischer Probleme, Krisen oder Erkrankungen bedeutet eine enorme Herausforderung für die psychiatrisch-psychotherapeutische Behandlung von Migranten (vgl. Assion 2005).

Gesichtspunkte der Rehabilitation und der Nachsorge von Persönlichkeitsstörungen bei Patienten mit interkulturellem Hintergrund sind bisher kaum wissenschaftlich untersucht. Eine Studie aus Spanien stellte allerdings fest, dass Immigranten und allgemein Personen, die primär wegen Persönlichkeitsstörungen in stationäre psychiatrische Behandlung kommen, eine geringere psychiatrische Behandlungskontinuität aufweisen (Gotor u. González-Juárez 2004). Möglicherweise muss bei Migranten mit Persönlichkeitsstörungen daher mit additiven Effekten gerechnet werden, was zu einer besonders schlech- 
ten Therapieadhärenz führen könnte. Hierzu liegen bis dato aber keine aussagekräftigen Daten aus wissenschaftlichen Untersuchungen vor.

\section{Zusammenfassung}

Die Abweichung einer Persönlichkeit von einer sozial akzeptierten Norm kann nur unter Berücksichtigung der sozialen Strukturen der Gesellschaft und der Werte, Vorstellungen und Normen der jeweiligen Kultur beurteilt werden. Bei Beachtung des kulturellen Kontextes sind der dimensionale Ansatz des Fünf-Faktoren-Modells, allgemeine Konzeptionen von Persönlichkeitsstörungen und vermutlich auch die Kriterien des DSM-IV mit ausreichender Reliabilität und Validität in sehr unterschiedlichen kulturellen Settings anwendbar.

Kulturelle Einflussfaktoren sind bei jedem Schritt des diagnostischen Prozesses von Persönlichkeitsstörungen bedeutsam. Auf die differenzialdiagnostisch abzugrenzenden Probleme durch eine Migration bzw. Akkulturation wird im DSM-IV explizit hingewiesen. Zusätzliche differenzialdiagnostische Schwierigkeiten können bei traumatisierten Migranten und Flüchtlingen auftreten, wenn psychische Traumafolgestörungen die Beurteilung einzelner Persönlichkeitsdimensionen erschweren. Die komplexen Zusammenhänge von Migration, Traumatisierung und Persönlichkeit bzw. Persönlichkeitsstörung entstehen in den einzelnen Phasen des Migrationsprozesses mit wechselseitigen Wirkrichtungen und kommen auf unterschiedlichen Zusammenhangsebenen zum Tragen.

Aus der Perspektive der interkulturellen Psychiatrie werden in den Prozessen der Migration und der Akkulturation sowie in den Folgen von prä- und postmigratorischer Traumatisierung wesentliche Stressoren bzw. Vulnerabilitätsfaktoren gesehen, die zu der Entwicklung oder zur Exazerbation einer Persönlichkeitsstörung beitragen oder deren Behandlung in der Praxis erschweren können.

\section{Literatur}

Alarcon R (1995) Culture and psychiatric diagnosis: impact on DSM-IV and ICD-10. Psychiatr Clin North Am 18, $449-465$

Alarcon R (1996) Personality disorders and culture in DSM-IV: a critique. J Pers Disord 10, 260-270

Alarcon R, Foulks E (1995) Personality disorders and culture: Contemporary clinical views, Part A: Cultural Diversity and Mental Health 1, 3-17

Alarcon RD, Foulks EF, Vakkur M (1998) Personality disorders and culture: Clinical and conceptual interactions. Wiley New York

Alarcon RD, Leetz KL (1998) Cultural intersections in the psychotherapy of borderline personality disorder. Am I Psychother 52, 176-190

Allik J, McCrae RR (2004) Toward a geography of personality traits. Patterns of profiles across 36 cultures. J Cross Cult Psych 35, 13-28

Al-Saffar S, Borgå P, Wicks S, Hällström T (2004) The influence of the patients' ethnicity, socio-demographic conditions and strain on psychiatric diagnoses given at an outpatient clinic. Nord J Psychiatry 58, 421-427

Amelang M, Bartussek D (1997) Differentielle Psychologie und Persönlichkeitsforschung. Verlag W. Kohlhammer Stuttgart

American Psychiatric Association (APA, 2000) Diagnostic and Statistical Manual of Mental Disorders: Text revision (4th ed.). American Psychiatric Association Washington D.C. 
Assion HJ (2005) Migration und psychische Krankheit. In: Assion H-J (Hrsg.) Migration und seelische Gesundheit. 133-144. Springer Berlin Heidelberg

Bandelow B, Schmahl C, Falkai P, Wedekind D (2010) Borderline personality disorder: a dysregulation of the endogenous opioid system? Psychol Rev 117, 623-636

Battan JF (1983) The „new narcissism“ in 20th century America: The shadow and substance of social change. I Soc Hist 17, 199-220

Briken P, Kraus C (2000) Persönlichkeitsstörungen. In: Haasen C, Yagdiran 0 (Hrsg.) Beurteilung psychischer Störungen in einer multikulturellen Gesellschaft. 125-144. Lambertus-Verlag Freiburg

Bronisch T (2001) Neurobiologie der Persönlichkeitsstörungen mit dem Schwerpunkt Borderline-Persönlichkeitsstörungen. Psychother 2, 233-246

Brucks U (2004) Der blinde Fleck der medizinischen Versorgung in Deutschland. Migration und psychische Erkrankung. Psychoneuro 30, 228-231

Brune M (2000) Posttraumatische Störungen. In: Haasen C, Yagdiran 0 (Hrsg.) Beurteilung psychischer Störungen in einer multikulturellen Gesellschaft. 107-124. Lambertus-Verlag Freiburg

Caldwell-Harris CL, Ayçiçegi A (2006) When personality and culture clash: the psychological distress of allocentrics in an individualist culture and idiocentrics in a collectivist culture. Transcult Psychiatry 43, 331-361

Calliess IT, Machleidt W, Ziegenbein M, Haltenhof H (2007) Suizidalität im Kulturvergleich. Fortschr Neurol Psychiat 75, 653-664

Calliess IT, Sieberer M, Machleidt W, Ziegenbein M (2008) Personality disorders in a cross-cultural perspective: impact of culture and migration on diagnosis and etiological aspects. Current Psychiatry Reviews 4, 39-47

Castaneda R, Franco H (1985) Sex and ethnic distribution of borderline personality disorder in an inpatient sample. Am I Psychiatry 142, 1202-1203

Chang SC (1995) The cultural context of Japanese psychiatry and psychotherapy. Am I Psychother 19, 593-606

Chavira DA, Grilo CM, Shea MT, Yen S, Gunderson IG, Morey LC, Skodol AE, Stout RL, Zanarini MC, McGlashan TH (2003) Ethnicity and four personality disorders. Compr Psychiatry 44, 483-491

Church AT (2000) Culture and personality: toward an integrated cultural trait psychology. I Pers 68, 651-703

Church AT (2001) Personality measurement in cross-cultural perspective. J Pers 69, 979-1006

Compton W, Helzer J, Hwu HG, Yeh EK, McEvoy L, Tipp J, Spitznagel E (1991) New methods in cross-cultural psychiatry: Psychiatric illness in Taiwan and the US. Am I Psychiatr 148, 1697-1704

Cooke DI (1996) Psychopathic personality in different cultures: What do we know? What do we need to find out? | Pers Disord 10, 23-40

Cooke DJ, Michie C (1999) Psychopathy across cultures: North America and Scotland compared. I Abnorm Psychol 108, 58-68

Costa PT, McCrae RR, Jonsson FH (2002) Validity and utility of the revised NEO personality inventory: examples from Europe. In: de Raad B, Perugini M (Hrsg.) Big Five Assessment. 61-77. Hogrefe u. Huber Publishers Seattle

DeGregorio E, Carver CS (1980) Type A behaviour pattern, sex role orientation and psychological adjustment. I Personal Soc Psychol Rev 39, 286-293

Distel MA, Rebollo-Mesa I, Willemsen G, Derom CA, Trull T, Martin NG, Boomsma DI (2009) Familial resemblance of borderline personality disorder features: genetic or cultural transmission? PLoS One 4(4), e5334

Distel MA, Trull TJ, Derom CA, Thiery EW, Grimmer MA, Martin NG, Willemsen G, Boomsma DI (2008) Heritability of borderline personality disorder features is similar across three countries. Psychol Med 38, 1219-1229

Fabrega H (1994) International systems of diagnosis. Psychiatry 182, 256-263

Fernando S (1991) Mental Health, Race and Culture. Macmillan Press Houndmills London

Fernando S (1995) Mental Health in a Multi-ethnic Society. Routledge London New York

Fiedler P (1998) Persönlichkeitsstörungen. Psychologie Verlags Union Weinheim

Foulks EF (1996) Culture and personality disorders. In: Mezzich JE, Kleinman A, Fabrega H, Parron DL (Hrsg.) Culture and psychiatric diagnosis: a DSM-IV perspective. 243-252. American Psychiatric Press Washington D.C.

Goldberg LR (1981) Language and individual differences: the search for universals in personality lexicons. Pers Soc Psychol Rev 2, 141-165 
Gotor L, González-Juárez C (2004) Psychiatric hospitalization and continuity of care in immigrants treated in Madrid (Spain). Soc Psychiatry Psychiatr Epidemiol 39, 560-568

Gunderson IG, Sabo AN (1993) The phenomenological and conceptual interface between borderline personality disorder and PTSD. Am | Psychiatry 150, 19-27

Huang CD, Church AT, Katigbak MS (1997) Identifying cultural differences in items and traits. Differential item functioning in the NEO personality inventory. I Cross Cult Psychol 28, 192-218

Igel U, Brähler E, Grande G (2010) Der Einfluss von Diskriminierungserfahrungen auf die Gesundheit von Migrantinnen. Psychiatr Prax 37, 183-190

Ikuta N, Zanarini MC, Minakawa K, Miyake Y, Moriya N, Nishizono-Maher A (1994) Comparison of American and Japanese outpatients with borderline personality disorder. Compr Psychiatry 35, 382-385

Johnson F (1993) Dependency and Japanese socialization. New York Universities Press New York

Kirmayer LJ (1989) Cultural variations in the response to psychiatric disorders and emotional distress. Soc Sci Med 29, 327-339

Kirmayer L L (2007) Psychotherapy and the cultural concept of the person. Transcult Psychiatry 44, 232-257

Krause IB (1998) Therapy Across Culture. Sage London

Lee S (1996) Cultures in psychiatric nosology: The CCMD-2-R and international classification of mental disorders. Cult Med Psychiatry 20, 421-472

Lewis-Fernandez $R$ (1993) The role of culture in the configuration of dissociative states: A comparison of Puerto Rican ataque de nervios and Indian possession syndrome. In: Spiegel D (Hrsg.) Dissociation, culture, mind and body. American Psychiatric Press Washington D.C.

Loranger AW, Sartorius N, Andreoli A, Berger P, Buchheim P, Channabasavanna SM, Coid B, Dahl A, Diekstra RF, Ferguson B, Jacobsberg LB, Mombour W, Pull C, Ono Y, Regier DA (1994) The International Personality Disorder Examination. The World Health Organization/Alcohol, Drug Abuse, and Mental Health Administration international pilot study of personality disorders. Arch Gen Psychiatry 51, 215-224

Machleidt W (2007) Die „kulturelle Adoleszenz“ als Integrationsleistung im Migrationsprozess. Psychotherapie und Sozialwissenschaft 9, 13-23

Machleidt W (2009) Wesentliche Merkmale interkultureller Psychotherapie. 59. Lindauer Psychotherapiewochen 2009, Lindau. URL: http://www.lptw.de/archiv/vortrag/2009/machleidt.pdf (abgerufen am 22.07.2014)

Machleidt W, Calliess IT (2005) Transkulturelle Psychiatrie und Migration - Psychische Erkrankungen aus ethnischer Sicht. Die Psychiatrie 2, 77-84

Machleidt W, Heinz A (2008) Psychotherapie bei Menschen mit Migrationshintergrund. In: Herpertz SC, Casper F, Mundt Ch (Hrsg.) Störungsorientierte Psychotherapie.627-637. Elsevier Urban u. Fischer München Jena

Markus HR, Kitayama S (1998) The cultural psychology of personality. I Cross Cult Psychol 29, 63-87

Martinez C (1993) Psychiatric care of Mexican Americans. In: Gaw AC (Hrsg.) Culture, ethnicity and mental illness. American Psychiatric Press Washington D.C.

McCrae RR (2001) Trait psychology and culture: exploring intercultural comparisons. I Pers 69, 819-846

McCrae RR (2002) NEO-PI-R data from 36 cultures: Further intercultural comparisons. In: McCrae RR, Allik I (Hrsg.) The five-factor model of personality across cultures. 105-125. Kluwer Academic/Plenum Publishers New York

McCrae RR, Costa PT (1987) Validation of the Five-Factor Model of personality across instruments and observers. I Pers Soc Psychol 52, 81-90

McCrae RR, Costa PT, Pilar GH, Rolland JP, Parker WD (1998) Cross-cultural assessment of the five-factor model. The revised Neo Personality Inventory. I Cross Cult Psychol 29, 171-188

McGilloway A, Hall RE, Lee T, Bhui KS (2010) A systematic review of personality disorder, race and ethnicity: prevalence, aetiology and treatment. BMC Psychiatry 2010; 10:33. URL: http://www.biomedcentral.com/ 1471-244X/10/33 (abgerufen am 22.07.2014)

Mezzich JE (1995) Cultural formulation and comprehensive diagnosis: Clinical and research perspectives. Psych Clinics of North Am 18, 649-657

Mezzich JE, Berganza CE, Ruiperez MA (2001) Culture in DSM-IV, ICD-10 and Evolving Diagnostic Systems. Psychiatr Clin North Am 24, 407-419 
Millon T (1993) Borderline personality disorder: a psychosocial epidemic. In: Paris I (Hrsg.) Borderline Personality Disorder: Etiology and Treatment. 197-210. American Psychiatric Press Washington D.C.

Millon T (2000) Sociocultural conceptions of the borderline personality. Psychiatr Clin North Am 23, 123-136

Moriya N, Miyake J, Minakawa K, Ikuta N, Nishizono-Maher A (1993) Diagnosis and clinical features of borderline personality disorder in the East and West: A preliminary report. Compr Psychiatry 34, 418-423

Murphy I (1976) Psychiatric labeling in cross cultural perspectives. Science 19, 1019-1028

Nestadt G, Samuels JF, Romanowski A, Folstein MF, McHugh PR (1994) DSM-III personality disorders in the community. Am | Psychiatry 151, 1055-1062

Nika L, Baskedis R (2000) Somatisierung. In: Haasen C, Yagdiran 0 (Hrsg.) Beurteilung psychischer Störungen in einer multikulturellen Gesellschaft. 71-88. Lambertus Freiburg

Padilla AM, Salgado-Snyder N, Cervantes RC (1987) Self-regulation and risk-taking behaviour: A Hispanic perspective. Spanish-Speaking Mental Health Research Center Bulletin, 1-5

Paris I (1991) Personality disorders, parasuicide and culture. Transcultural Psychiatric Research Review 28, 25-39

Paris I (1992) Social factors in borderline personality: A review and hypothesis. Can | Psychiatry 37, 480-486

Paris I (1996) Cultural factors in the emergence of borderline pathology. Psychiatry 59, 185-192

Paris I (1998) Personality disorders in sociocultural perspective. | Pers Disord 12, 289-301

Pascual IC, Malagón A, Córcoles D, Ginés JM, Soler J, García-Ribera C, Pérez V, Bulbena A (2008) Immigrants and borderline personality disorder at a psychiatric emergency service. Br I Psychiatry 193, 471-476

Poortinga YH, Van Hemert DA (2001) Personality and culture: demarcating between the common and the unique. / Pers 69, 1033-1060

Robins LN, Tipp I, Przybeck T (1991) Antisocial personality disorder. In: Robins LN, Regier DA (Hrsg.) Psychiatric disorders in America. 258-290. Free Press New York

Roland A (1988) In search of self in India and Japan. Princeton University Press Princeton

Sieberer M, Ziegenbein M, Eckhardt G, Machleidt W, Calliess IT (2011) Psychiatrische Begutachtung im Asylverfahren. Psychiat Prax 38, 38-44

Standage K, Bilsbury C, Jain S, Smith D (1984) An investigation of role-taking in histrionic personalities. Can J Psychiatry 29, 407-411

Tang SW, Huang Y (1995) Diagnosing personality disorders in China. International Medical Journal 2, 291-297

Trouve IN, Lianger JP, Colvet P, Scotto IC (1983) Sociological aspects of identity problems in immigration pathology. Annals Medico-Psychologiques 141, 1041-1062

Tseng WS (2001) Handbook of Cultural Psychiatry. Academic Press San Diego London Boston New York Sydney Tokyo Toronto

Tuna S, Salman R (1999) Phänomene interkultureller Kommunikation im Begutachtungsprozess. In: Collatz I, Hackhausen W, Salman R (Hrsg.) Begutachtung im interkulturellen Feld. Zur Lage der Migranten und zur Qualität ihrer sozialgerichtlichen und sozialmedizinischen Begutachtung in Deutschland. 179-188. VWB Verlag für Wissenschaft und Bildung Berlin

Van de Vijver FI, Leung K (2001) Personality in cultural context: methodological issues. I Pers 69, 1007-1031

Witztum E, Greenberg D, Dasberg H (1990) Mental illness and religious change. British Journal of Medicine and Psychology 63, 33-41

Yang I, McCrae RR, Costa PT, Yao S, Dai X, Cai T, Gao B (2000) The cross-cultural generalizability of Axis-II constructs: an evaluation of two personality disorder assessment instruments in the People's Republic of China. I Pers Disord 14, 249-263

Zhong I, Leung F (2009) Diagnosis of borderline personality disorder in China: current status and future directions. Curr Psychiatry Rep 11, 69-73 